\title{
Hydroacoustic noise from different geometries
}

\author{
M. Cianferra*,a, V. Armenio ${ }^{\mathrm{a}}, \mathrm{S}$. Ianniello ${ }^{\mathrm{b}}$

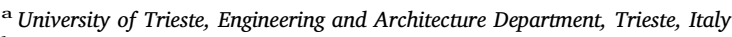 \\ ${ }^{\mathrm{b}}$ CNR-INSEAN, Marine Technology Research Institute, Rome, Italy
}

A R T I C L E I N F O

\section{Keywords:}

Hydroacoustic

Ffowcs Williams and Hawkings analogy LES

Turbulent flows

\begin{abstract}
A B S T R A C T
Turbulent flow around bluff bodies generates pressure fluctuations which propagate as acoustic waves Differences in the shape of a body can affect frequencies and amplitudes of the propagating pressure signals. In the present work three elementary geometries (sphere, cube and prolate spheroid), immersed in a uniform water flow, are examined in order to analyze the differences of the resulting hydroacoustic fields. The turbulent flow at $R e_{A}=4430$ (based on the cross-sectional area of the bodies) is reproduced through wall-resolving Large-Eddy Simulation and the hydroacoustic far-field is analyzed by adopting the Ffowcs Williams and Hawkings analogy. The quadrupole term of the acoustic equation is first reformulated in the convective form and then solved through direct computation of the volume integrals. This procedure is found possible in hydrodynamics where the speed of sound is very large and the flow velocities are small. In spite of the fact that the frontal section of the bodies has the same area, the analysis shows that a streamlined body is able to produce a pressure signal one order of magnitude lower than that generated by a bluff geometry. The separate analysis of the loading noise and of the quadrupole one has shown that the former is larger than the latter in case of 3D-shaped bluff body (sphere and cube), whereas the opposite is true in case of a streamlined body. A preliminary analysis between the case of an elongated square cylinder and a cube, shows that the persistence of a two-dimensionally shaped wake when compared to a three-dimensional one contributes to increase the quadrupole part of the radiated noise.
\end{abstract}

\section{Introduction}

Fluid dynamic noise constitutes a serious issue in a number of engineering applications and growing attention is being paid toward new generation mathematical models able to perform reliable noise predictions (see, among the others, Carlton and Vlasic, 2005 and Murphy and King, 2014).

Since sound represents propagation of pressure/density disturbances, in principle the Navier-Stokes equations for compressible flows should be solved for the study of near and far-field sound propagation (this is known in literature as direct method). However, few studies of this kind are available in literature, mostly limited to 2D cases or elementary configurations (see, for example, Inoue and Hatakeyama, 2002; Marsden et al., 2008), because the use of a direct method may be unpractical for two main reasons. When the fluid-dynamic field is incompressible (Mach number smaller than 0.3 and, in general, all main problems concerning the generation and propagation of noise underwater), the problem is substantially elliptic, and the use of numerical methods suited for hyperbolic problems (like the compressible flow field) may produce an ill-conditioned system of equations whose numerical solution is practically impossible; second, the computational domain normally used for a computational fluid dynamic (CFD) solution is necessarily limited in size and much smaller than the distance where the knowledge of the hydrodynamic noise is usually required. To overcome these problems, hybrid methods have been developed in the past (mainly for aeronautical configurations) and nowadays they constitute the standard numerical approach in the acoustic community. The hybrid method allows to decouple the fluid dynamic problem from the acoustic one. The fluid dynamic field is determined using CFD solutions obtained in the flow regime of interest (either incompressible or compressible) within a suitable computational domain. The acoustic field is obtained using an acoustic analogy, where the conservation laws are re-written as an inhomogeneous wave equation and the flow is treated as a collection of noise sources. The coupling between the fluid dynamic part of the problem and the acoustic one is carried out using the instantaneous fields obtained in the CFD solution as input data for the acoustic equation. The most important advantage of the hybrid method stands in the fact that, starting from a confined fluid dynamic domain, the acoustic solution can be projected onto the far field, at any point of interest. Further, due to the presence of different source terms, the inhomogeneous wave equation provides a simple identification of the dominant source mechanisms taking place in the flow.

\footnotetext{
* Corresponding author.

E-mail addresses: marta.cianferra@phd.units.it (M. Cianferra), vincenzo.armenio@dia.units.it (V. Armenio), sandro.ianniello@cnr.it (S. Ianniello).
} 
Different formulations of the equations for the acoustic field have been developed in literature. In Ffowcs-Williams and Hawkings (1969), a comprehensive formulation was given (hereafter referred to as FW-H equation) in which a body in relative motion with respect to the surrounding fluid can be considered.

The integral form of the FW-H equation consists of a sum of surface and volume integrals, which are commonly identified as dipole and quadrupole terms respectively. In literature, the direct evaluation of the quadrupole noise terms has rarely been carried out for two main reasons: It is considered very expensive from a computational point of view; it may be affected by computational noise in case of sharp discontinuities in the pressure/density field occurring in the fluid dynamic compressible regime. In addition, in the aeroacoustic literature (such as in studies of helicopter noise), the non-linear quadrupole terms are usually considered negligible in comparison to the linear ones, namely, the loading noise associated to the presence of a body and the thickness noise related to its own movement.

However, in their fundamental work (see for example Farassat and Brentner, 1988; Farassat and Brentner, 2003), the authors pointed out the significant role played by the quadrupole terms in the radiated noise. In particular, they reformulated it for three different regions (boundary layer, shock surfaces and tip vorticity/wake) in such a way to obtain surface integrals instead of volume integrals. They provided detailed considerations on the fact that the quadrupole noise may behave as thickness and loading noise and, for example, suggested that the tip vorticity effect can be converted into a line integral along the vortex line and that the blade wake contribution can be written in such a way that only the gradient of velocity normal to the wake appears. Further, the authors emphasized the need of using accurate fluid-dynamic data and fine spatial resolution for a reliable reconstruction of the radiated noise.

For all reasons mentioned above, the quadrupole noise is generally formulated through an alternative approach, known as porous formulation (see DiFrancescantonio, 1997). This method consists in moving the surface integrals from the body surface over an external porous radiating surface, embedding the body and the whole fluid region characterized by nonlinear sources. In Cianferra et al. (2017) a comparison between the porous method and the direct evaluation of the quadrupole terms was carried out. When the porous method is applied without corrections that eliminate the end-cap problem (namely the spurious noise generated by vorticity crossing the porous surface, see Nitzkorski and Mahesh, 2014) the direct computation of the volume terms provides the most reliable and accurate results. However, the authors also pointed out that the direct evaluation of volume integrals is feasible from a computational point of view when times delays are negligible, that is, when a collection of noise sources can be considered as to propagate instantaneously (this concept will be exploited in the next Section). In general, a motionless/slowly moving body immersed in a stream of water belongs to this case, thus, in most cases in hydrodynamics, the source noise can be assumed compact.

In the recent years, the acoustic analogy has been used in several applications in literature. Among the others, studies of realistic geometries for marine applications were carried out by Ianniello et al. (2013), Li et al. (2015), Lidtke et al. (2015, 2016) and Bensow and Liefvendahl (2016). These works focused on the underwater propeller noise, employing the $\mathrm{FW}-\mathrm{H}$ porous formulation in conjunction with fluid dynamic fields obtained solving the Unsteady Reynolds Averaged Navier-Stokes (URANS) equations or using Detached Eddy Simulation (DES).

More fundamental studies, focused on hydrodynamic noise generated by simple-shape objects, were carried out by, among the others, Lockard et al. (2007), Pando et al. (2014) and Gloerfelt et al. (2005). Specifically, Lockard et al. (2007) performed experimental and numerical (URANS) studies of a tandem cylinder configuration. Extensive comparisons with the experimental data were carried out to assess the ability of the computations to simulate the details of the flow and the radiated noise. Their acoustic analysis was based on the method described in Lockard (2002), where the author compared a frequency domain solution method of the FW-H equation with the (porous) retarded-time formulation.

Pando et al. (2014) performed direct numerical simulations of the compressible Navier-Stokes equations and showed good agreement with previous experimental and numerical investigations on noise radiated from a NACA0012 airfoil.

Gloerfelt et al. (2005) studied the flow around a circular cylinder. Curles formulation was analytically and numerically compared to a formulation based on an exact Greens function tailored to a cylindrical geometry.

In spite of the geometrical simplification, the study of hydrodynamic noise generated around simple bodies has proved to be significant, because it can exploit fundamental aspects of the topology of the flow field which, in turn, rules generation and propagation of hydrodynamic noise.

To the best of our knowledge, a systematic study of noise generated by elementary geometries different from those mentioned above, has not been carried out, especially for underwater problems. In the present paper, we give a contribution to this aspect, considering three simple, yet significant, geometries in the turbulent regime: A sphere, a cube and a prolate spheroid at zero angle of attack. The sphere produces massive separation at the rear of the body and a wake characterized by overlapping of vortex shedding and energetic turbulence generated by a shear layer; the cube behaves likewise the sphere, apart the presence of sharp corners which may contribute to noise generation; the prolate spheroid aligned with the main current, develops a small separation region in the trailing edge region and a wake much less intense than in the other cases. Finally, a preliminary comparison between the noise generated by the cube and that given by a 2D-shaped geometry (the elongated square cylinder studied in Cianferra et al., 2017) is carried out, to evaluate the contribution of the nonlinear term to the far-field noise propagation in case of $3 \mathrm{D}$ and $2 \mathrm{D}$ massive separation respectively.

For sake of comparison, the Reynolds number, based on the square root of the frontal area, the uniform inlet velocity, and viscosity, is $R e_{A}=\sqrt{A} U_{0} / \nu=4430$ for the three objects. The fluid dynamic field is solved using wall-resolving Large Eddy simulation (LES), able to reproduce the energetic part of the energy spectrum, which mostly contributes to the radiated noise (see Piomelli et al. (1997) and Seror et al. (2000)). The acoustic field is reconstructed by using the FW$\mathrm{H}$ equation, computing the non-linear quadrupole terms through direct volume integration. The main contributions of the present paper to the literature are: Evaluation of the far-field noise for three significant geometries; application of the direct volume method to the evaluation of the quadrupole term; a preliminary estimation of the contribution of 2D- and 3D-shaped wakes to the far-field noise; wall-resolving LESgenerated database of fluid-dynamic data available to the scientific community for successive studies.

The paper is organized as follows. Section 2 provides a concise theoretical background, for both fluid dynamic (Section 2.1) and acoustic (Section 2.2) models; Section 3 contains the general features of the numerical setup. Section 4 contains: Validation of the results for the fluid dynamic field on the sphere together with a test on the acoustic model adopted (Section 4.1); a comparison of the acoustic far-field generated by the three different objects (Section 4.2); a comparison of radiated noise in case of 2D- and 3D-shaped wakes (Section 4.3). Concluding remarks are given in Section 5 .

\section{The mathematical formulations}

Both fluid dynamic and acoustic models are based on the 
Navier-Stokes equations. The present paper deals with single-phase flow conditions (no cavitation) in the incompressible regime. Under these conditions, since the compressibility nature of sound propagation is completely described by the FW-H wave equation, the incompressible flow assumption can be adopted to solve the underlying fluid dynamic problem.

\subsection{Fluid dynamic model}

We use LES, in which the large anisotropic and energy-carrying scales of motion are directly resolved through an unsteady and 3D simulation, whereas the more isotropic and dissipative small scales of motion are confined in the sub-grid space. Scale separation is carried out through a filtering operation of the flow variables. As discussed in the Introduction, in literature the contribution of the subgrid scales (SGS) of motion on noise generation and propagation has been found negligible. The constant-density filtered Navier-Stokes equations in the incompressible regime read as:

$\frac{\partial \bar{u}_{i}}{\partial x_{i}}=0$,

$\frac{\partial \bar{u}_{i}}{\partial t}+\frac{\partial \bar{u}_{i} \bar{u}_{j}}{\partial x_{j}}=-\frac{1}{\rho_{0}} \frac{\partial \bar{p}}{\partial x_{i}}+\nu \frac{\partial^{2} \bar{u}_{i}}{\partial x_{j} \partial x_{j}}-\frac{\partial \tau_{i j}^{s g s}}{\partial x_{j}}$,

where the overbar denotes the filtering operation, $u_{i}$ is the velocity component in the $x_{i}$ direction, $p$ the hydrodynamic pressure and $\rho_{0}$ the fluid density. Here we use interchangeably $u_{1}, u_{2}, u_{3}$ or $u, v, w$ as velocity components along the $x_{1}, x_{2}, x_{3}$ or $x, y, z$ directions respectively. The SGS stress tensor $\tau_{i j}^{s g s}=\overline{u_{i} u_{j}}-\bar{u}_{i} \bar{u}_{j}$ represents the effect of the unresolved fluctuations on the resolved motion. They need to be modeled and in the present work we use the dynamic Smagorinsky eddy-viscosity model:

$\tau_{i j}^{s g s}-\frac{1}{3} \tau_{k k}^{s g s} \delta_{i j}=-2 v_{t} \bar{S}_{i j}$, in which $\bar{S}_{i j}=\frac{1}{2}\left(\frac{\partial \bar{u}_{j}}{\partial x_{i}}+\frac{\partial \bar{u}_{i}}{\partial x_{j}}\right)$

and $\delta_{i j}$ is the isotropic second order tensor. The SGS Eddy viscosity $\nu_{t}$ is expressed as:

$v_{t}=\left(C_{s} \Delta\right)^{2}\left|\bar{S}_{i j}\right|$,

where $\Delta=\sqrt[3]{\Delta x_{1} \Delta x_{2} \Delta x_{3}}$ is the filter width and the constant $C_{s}$ is determined using the dynamic Lagrangian procedure of Meneveau et al. (1996).

\subsection{Acoustic model}

Assuming small perturbations, after linearization and manipulation of the compressible Navier-Stokes equations, it is possible to achieve a wave equation characterizing the propagation of the acoustic pressure. This pressure originates in the flow field and propagates in the far-field where the medium is assumed quiescent. The FW-H equation accounts for the presence of a rigid body moving in the fluid and treats it as a discontinuity. In our case, the body as well as the observer are at rest and immersed in a uniform flow, so that the terms related to body velocity drop out. Nevertheless, the surrounding fluid moves at a constant speed (along the $x$ axis), so that the acoustic formulation is derived from the advective form of the governing equation.

A clear derivation of the advective FW-H equation is reported in the paper of Najafi-Yazdi et al. (2011), where the authors developed an integral solving formulation for the linear (surface) terms, validated it for a monopole (stationary and rotating) and a dipole in a moving medium. The authors also gave a detailed description of the "wind-tunnel" problem herein investigated, where both the source and the observer are at rest. Note that in that paper (as well as in most papers focused on the use of the FW-H equation), the nonlinear volume term was not included in the solving formulation, since the authors assumed its contribution to be negligible or, if needed, assessable through the porous formulation. Here we report the advective formulation of the volume term for the particular case of the wind tunnel flow, by using the free-space Green's function for the advective wave equation (see Blokhintsev, 1956). The acoustic pressure $\widehat{p}$, at any point $\mathbf{x}$ and time $t$, is represented by the sum of surface () and volume $\left(\widehat{p}_{3 D}\right)$ integrals, respectively:

$$
\begin{aligned}
4 \pi \hat{p}_{2 D}(\mathbf{x}, t)=\frac{1}{c_{0}} & \frac{\partial}{\partial t} \int_{S}\left[\frac{\widetilde{p} \hat{n}_{i} \hat{r}_{i}}{r^{*}}\right]_{\tau} d S+\int_{S}\left[\frac{\widetilde{p} \hat{n}_{i} \hat{r}_{i}^{*}}{r^{* 2}}\right]_{\tau} d S \\
4 \pi \hat{p}_{3 D}(\mathbf{x}, t)=\frac{1}{c_{0}^{2}} & \frac{\partial^{2}}{\partial t^{2}} \int_{f>0}\left\{T_{i j}\left[\frac{\hat{r} \hat{r}_{j}}{r^{*}}\right]\right\}_{\tau} d V \\
& +\frac{1}{c_{0}} \frac{\partial}{\partial t} \int_{f>0}\left\{T_{i j}\left[\frac{2 \hat{r}_{i} \hat{r}_{j}^{*}}{r^{* 2}}+\frac{\hat{r}_{i}^{*} \hat{r}_{j}^{*}-R_{i j}^{*}}{\beta^{2} r^{* 2}}\right]\right\}_{\tau} d V \\
& +\int_{f>0}\left\{T_{i j}\left[\frac{3 \hat{r}_{i}^{*} \hat{r}_{j}^{*}-R_{i j}^{*}}{r^{* 3}}\right]\right\}_{\tau} d V .
\end{aligned}
$$

$\widetilde{p}=p-p_{0}$ is the pressure perturbation with respect to the reference value $p_{0}, \hat{n}$ is the (outward) unit normal vector to the surface element $d S, \hat{r}$ and $\hat{r}^{*}$ are unit radiation vectors (see Appendix A), $r$ and $r^{*}$ are the module of the radiation vectors $\mathbf{r}$ and $\mathbf{r}^{*}$ respectively and $c_{0}$ is the sound speed. Eq. (4) contains two second-order tensors: $R_{i j}^{*}$ and the Lighthill stress tensor $T_{i j}$, the latter characterizing the FW-H quadrupole term. Under the assumption of negligible viscous effects and isoentropic transformations for the fluid in the acoustic field the Lighthill tensor reads as:

$T_{i j}=\rho_{0} u_{i} u_{j}+\left(\widetilde{p}-c_{0}^{2} \widetilde{\rho}\right) \delta_{i j}$,

where $\widetilde{\rho}$ is the density perturbation of the flow and in our case is zero. The surface integrals in Eq. (3) are referred to as linear terms of the FW$\mathrm{H}$ equation and represent the loading noise term. The volume integrals in Eq. (4) are slightly different from the standard FW-H (non-advective) equation. For their derivation we consider a uniform flow with velocity $U_{0}$ along the direction $x_{1}$.

Obviously, the direct integration of the volume terms gives accurate results, however this method can be used if the calculation of the time delays (discussed below) can be omitted, otherwise the computational burden makes it unfeasible. The calculation of the time delays, in fact, requires to store at each time step pressure and velocity data related to the entire (noise-source) volume, in order to perform an interpolation over all the data.

However, for the cases herein investigated of compact noise source, we can make the following observation: If a microphone is located, for example, at $\mathbf{x}=(0,0,100 D)$ (the origin of the frame of reference is at the center of the body), the maximum time delay is

$(t-\tau(\mathbf{y}))_{\max }=\max _{\mathbf{y} \in V}(\mathbf{x}-\mathbf{y}) / c_{0} \approx 109 D / c_{0}=7.7 \times 10^{-4}$

while the minimum time delay is $\approx 92 D / c_{0}=6.5 \times 10^{-4}$. The difference between them is $d \tau \sim 1.2 \times 10^{-4}$. It means that pressure signals, coming from the flow (noise source), overlap if their emission occurs at time intervals $d t<d \tau$. In this case the signal coming from the source points farthest from the microphone will start overlapping the signal coming from the nearest source region, emitted in the successive time step thus creating a complex composition of single signal. Note that in the case herein investigated the time delay is very small and the composition of the signals is not expected to contribute to the radiated noise. Also, since the time step $d t$ at which we sampled the data for the acoustic analysis is much larger than $d \tau$ (of about a factor 10), the pressure signals emitted at every $d t$ reach the microphone location, in practice, simultaneously, and the evaluation of the single time delays may be 
reasonably omitted. This procedure allows for a remarkable saving of CPU time and makes the direct computation of the quadrupole volume terms feasible. Note that this holds for a number of cases in hydrodynamic applications. Conversely, in aerodynamic applications, where $c_{0}$ is much smaller than in the hydrodynamic case, $d \tau$ may be large, in particular when in presence of fast moving bodies (i.e. helicopter blades) and the computation of the time delays is not negligible.

As to perform a validation test for the acoustic model, we compare the solution of the advective FW-H equation with the pressure signal provided by LES, considered as reference data. The measurement points, in this procedure, are selected within the fluid dynamic domain. This comparison is useful to verify the ability of the acoustic post processing to reconstruct accurately the pressure field. Then, an analysis of the acoustic far-field related to the three different geometries is carried out, for different measurement locations.

\section{Numerical setup}

Both fluid dynamic and acoustic fields are solved in the framework of the OpenFOAM ${ }^{\circ}$ library, based on Finite Volume Methods (FVM). The filtered Navier-Stokes equations are integrated using the pisoFoam solver, with spatial derivatives discretized through second-order central differences. Implicit time advancement runs according to the Euler scheme. The algorithm, including the SGS closure, has been customized at the laboratory of Industrial and Environmental Fluid Mechanics (IEFluids) of the University of Trieste, and details can be found in Cintolesi et al. (2015).

The FW-H solving formulation was implemented as a post-processing utility. Here, for sake of completeness and self-consistence of the paper, we give a description of the procedure. The size and shape of the integration volume is given as input. The algorithm reads the flow data, (i.e. velocity and pressure fields), the surface of the body and the volume of the cells over which to calculate the integrals. It calculates the integrand functions of Eqs. (3) and (4) over each single element respectively and then it sums over the whole integration domains. The time derivatives appearing in Eqs. (3) and (4) were calculated using both second- and forth-order schemes. The differences in the quality of the results were found negligible.

At each noise source point of the integration domain, the emission time $\tau$ is fixed and identified by the time step of LES run, so that the corresponding observer time $t$ has to be determined by a data fitting algorithm. As previously discussed, due to the absence of body motion, to the high value of sound speed and to the small length scale of the body, the compressibility delay $t-\tau$ practically reduces to a time shift homogeneous in space, which does not affect the composition of elementary signals. In fact, after switching off the computation of the compressibility delay, so as to have practically instantaneous source propagation, we noted that results were practically identical. In other words, the body together with its own wake appears as a compact source and the calculation of the delays can be omitted. As discussed in the Introduction, this is widely accepted in hydroacoustics (see, among the others, Nitzkorski and Mahesh, 2014 for a discussion).

We consider three bodies, respectively a sphere, a cube and a 0 angle of attack prolate spheroid. The sphere diameter is $D=0.01 \mathrm{~m}$. It is immersed in a water stream with velocity $U_{0}=0.5 \mathrm{~m} / \mathrm{s}$ and kinematic viscosity $\nu=1.0 \times 10^{-6} \mathrm{~m}^{2} / \mathrm{s}$, so that the Reynolds number based on the sphere diameter $D$ is $R e_{D}=5000$. In order to compare the three different geometries, the Reynolds number based on the square root of the reference (frontal) area is the same for the three configurations $R e_{A}=\sqrt{A} U_{0} / \nu=4430$. The side of the cube is $l=0.008 \mathrm{~m}$, the minoraxis of the ellipsoid is equal to the sphere diameter $D$ and its major-axis is $6 D$.

The computational domain for the sphere and the cube is a box with dimensions $16 D \times 16 D \times 16 D$ along the $x, y$ and $z$ axes respectively.
The domain for the prolate spheroid is $24 D \times 16 D \times 16 D$ along the three directions. At the boundaries of the computational domain we set a zero-gradient condition for the pressure but at the outlet, where pressure is set to zero. The velocity is set to $U_{0}$ at the inlet, stress-free condition is set at the lateral boundaries and zero gradient condition is set for the velocity components at the outlet.

In order to evaluate the accuracy of the simulations, for the case of the sphere, for which available experimental and numerical reference data are available, two unstructured, body-fitted grids were used: A coarse one (CG) consisting of about 5 millions of cells and a fine one (FG) consisting of about 8 millions of cells. The meshes were built employing the utility of OpenFoam snappyHexMesh. In the CG case, the grid spacing normal to the wall for the first layer of cells is $0.001 D$ at the body surface directly resolving the viscous sub-layer; a refinement box around the body was considered so as to obtain, in the wake region, a grid size of about $0.1 D$ at a distance of $8 D$. Out of the region of interest a coarser grid allows the increase of the dimensions of the domain, reducing possible disturbance effects coming from the boundaries. An $A$ posteriori analysis showed that about 5 grid points where placed within 10 wall units $\left(y^{*}=\nu / u_{\tau}\right.$ with $u_{\tau}=\sqrt{\tau_{w} / \rho_{0}}$ and $\tau_{w}$ the mean shear stress) off the wall. In the FG case, same conditions were considered in the proximity of the body surface, hence the first layer of cells is $0.001 \mathrm{D}$, but a refinement was carried out in a rectangular region which embeds the sphere and its wake, up to $9 D$ downstream, so as to have a grid size less than $0.05 D$ at a distance of $8 D$, about 8 grid points within 10 wall units off the body and also smaller grid size in the plane parallel to the body surface.

A constant time step $\Delta t=5 \times 10^{-5} \mathrm{~s}$ was used for the CG case, whereas it was reduced to $\Delta t=10^{-5}$ for the FG case. In both cases, the Courant number was kept smaller than 0.5 . The flow around the sphere, after about 80 characteristic times $D / U_{0}$, was completely developed. After that, statistics were accumulated, collecting the flow data for additional $40 D / U_{0}$ (coarse grid) and $20 D / U_{0}$ (fine grid), sampled every $0.1 D / U_{0}$. The pressure and velocity fields were then employed for the acoustic analysis.

The analysis of the results obtained with the two grids and comparisons with reference data (reported in the next Section) show that the FG was able to give results more accurate than the CG.

For this reason, the cases of the cube and of the prolate spheroid were run using a fine grid of about 8 million cells, built in a way similar to the case of the sphere. For the flow around the cube, the flow data were collected every $0.25 \mathrm{D} / U_{0}$ over a period of $20 \mathrm{D} / U_{0}$; for the prolate spheroid data collection was performed every $0.2 D / U_{0}$ over a period of $35 D / U_{0}$.

The data obtained in the three cases were employed as input for the acoustic solver. The acoustic analogy was applied to determine the acoustic pressure time-history at different measurement points (named microphones or probes). As mentioned, calculations refer to water. For the acoustic field the fluid density is set to $1000 \mathrm{Kg} / \mathrm{m}^{3}$, while the speed of sound is equal to $1400 \mathrm{~m} / \mathrm{s}$.

\section{Results}

This section is composed of three subsections. The first one describes the main features of the turbulent flow around the sphere and validate our numerical results against the available literature data of Rodriguez et al. (2010), Kim and Durbin (1988) and Seidl et al. (1998). As mentioned, to test the adequacy of the mesh two different simulations were performed, one on the CG of about 5 million of cells and one on the FG of about 8 million of cells. The subsection also shows the validation of the acoustic solver using LES reference data for the sphere case.

The second subsections contains the analysis of the acoustic far-field produced by the three geometries, at different microphones. The third 
subsection contains a preliminary discussion on difference between 2Dand 3D-shaped wakes with respect to the far field noise.

\subsection{Validation of the fluid dynamic and acoustic solvers - sphere case}

The subcritical flow around a sphere undergoes laminar separation near the equator and exhibits an instability appearing as a progressive wave motion with alternate fluctuations produced by the shear at the edge of the separation present in the wake region. The large scale (sinuous) instability of the wake is commonly referred to as "vortex shedding", though measurements and smoke visualizations of (Kim and Durbin, 1988) showed no evidence of discrete vortex shedding behind the sphere. The mode associated with the small-scale shear-layer Kelvin-Helmholtz instability at the edge of the recirculation region, is responsible for the distortion of the large-vortex structures, for production of small scales and, eventually, for transition to turbulence in the detached shear layers.

Experiments clearly show that the two instability modes co-exist up to a certain Reynolds number in the subcritical regime, although there is disagreement on its actual value. Achenbach (1974) did not detect the high frequency mode beyond $R e=6000$; Sakamoto and Haniu (1990) did not observe it beyond $R e=1.5 \times 10^{4}$ whereas Chomaz et al. (1993) were able to capture it at $R e=3 \times 10^{4}$. The measurements of Kim and Durbin (1988) and Bakic (2002) showed the presence of the two modes up to $R e=10^{5}$.

The Strouhal number corresponding to the shedding spiral mode remains approximately in the range $0.18-0.2$. However, according to the experimental data of Achenbach (1974), in the range $R e=6 \times 10^{3}$ to $3 \times 10^{4}$, the Strouhal number rises from $S t=0.125$ to $S t=0.18$. In experimental studies, a higher frequency component is also observable, associated with the development of the Kelvin-Helmholtz instabilities in the detached shear layers, $(S t \geq 1.3)$. A numerical simulation may need a high level of grid refinement in the shear layer region to detect the small-scale instability, as in the DNS study of Seidl et al. (1998).

We compare our results obtained with two grids with the reference DNS data of Seidl et al. (1998) and the experimental data of Kim and
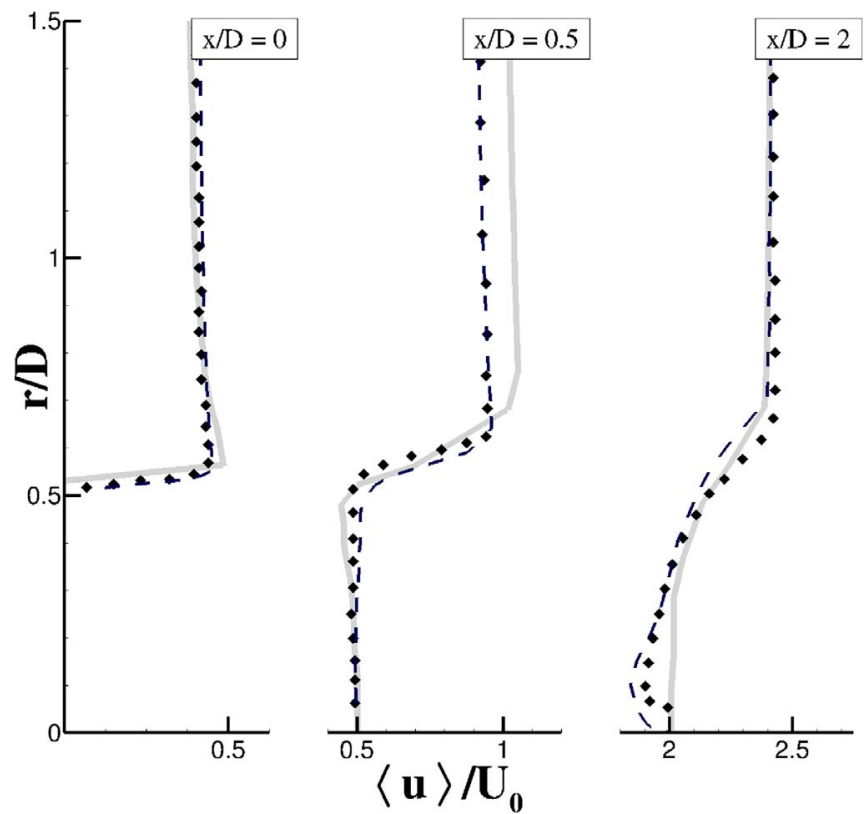

Fig. 1. Mean streamwise velocity (made nondimensional with $U_{0}$ ) along the radial direction at three different positions along the $x$-axis: $x=0, x=0.5 D$ and $x=2 D$. CG, solid line; FG, dashed line; DNS results of Seidl et al. (1998), dots. Note that, according to the notation used in Seidl et al. (1998), the radial direction runs in planes orthogonal to the $x$ axis and is centered at the symmetry line $y=0, z=0$, like in cylindrical coordinates.

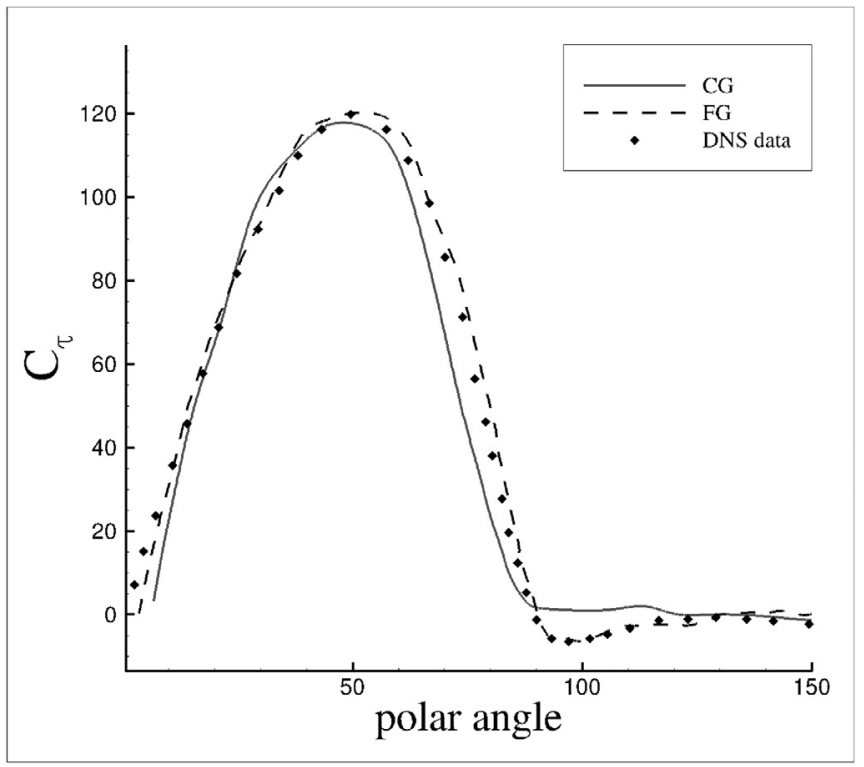

Fig. 2. Friction coefficient along the circumferential direction expressed in degrees ( 0 is the trailing edge, 90 is at the equator, 180 (not shown) is the trailing edge): CG, solid line; FG, dashed line; DNS results of Seidl et al. (1998), dots.

Durbin (1988), both at the same value of the Reynolds number as in our case.

Hereafter, quantities averaged in time and over a direction in space are denoted with the symbol $\langle$. $\rangle$. The mean streamwise velocity $\langle u\rangle$ (made non-dimensional with $U_{0}$ ) along the radial direction, is depicted at three different positions along the $x$-axis: $x=0, x=0.5 D$ and $x=2 D$ (Fig. 1). As in Seidl et al. (1998), the mean velocity profiles are calculated over the cross-stream planes $x=$ const averaging over the circumferential direction at constant distance from the axis of symmetry with coordinates $y=0, z=0$. Note that the radial direction depicted in the figure does not coincide with that one defined in spherical coordinates. The origin of the Cartesian frame of reference is at the center of the sphere, hence the plane $x=0$ corresponds to the equatorial plane of the sphere normal to the inlet velocity.

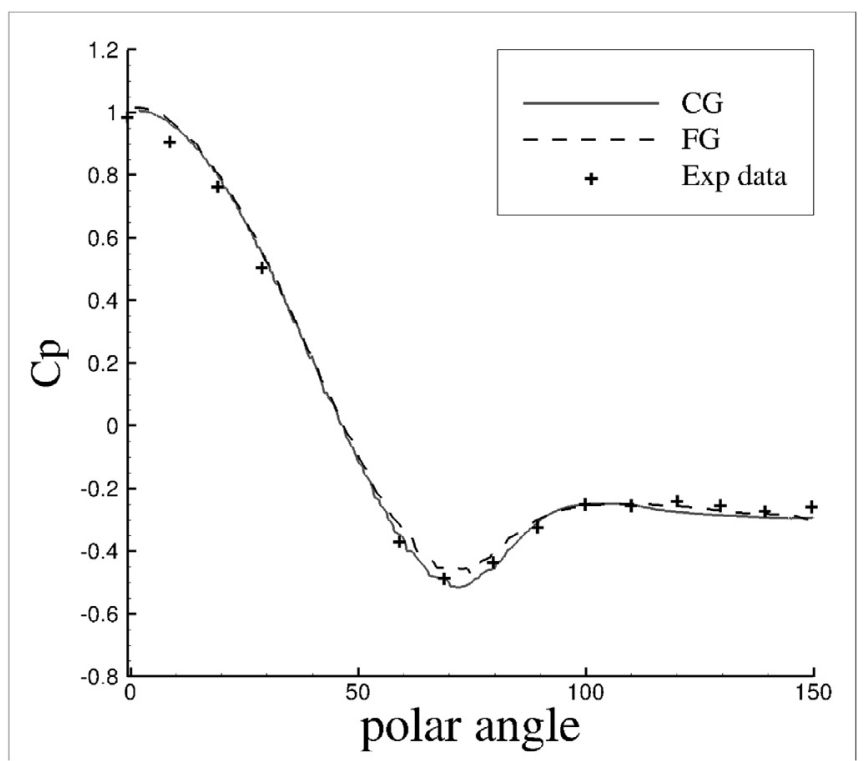

Fig. 3. Pressure coefficient along the circumferential direction expressed in degrees ( 0 is the trailing edge, 90 is at the equator, 180 (not shown) is the trailing edge): CG, solid line; FG, dashed line; experimental data of Kim and Durbin (1988), crosses. 


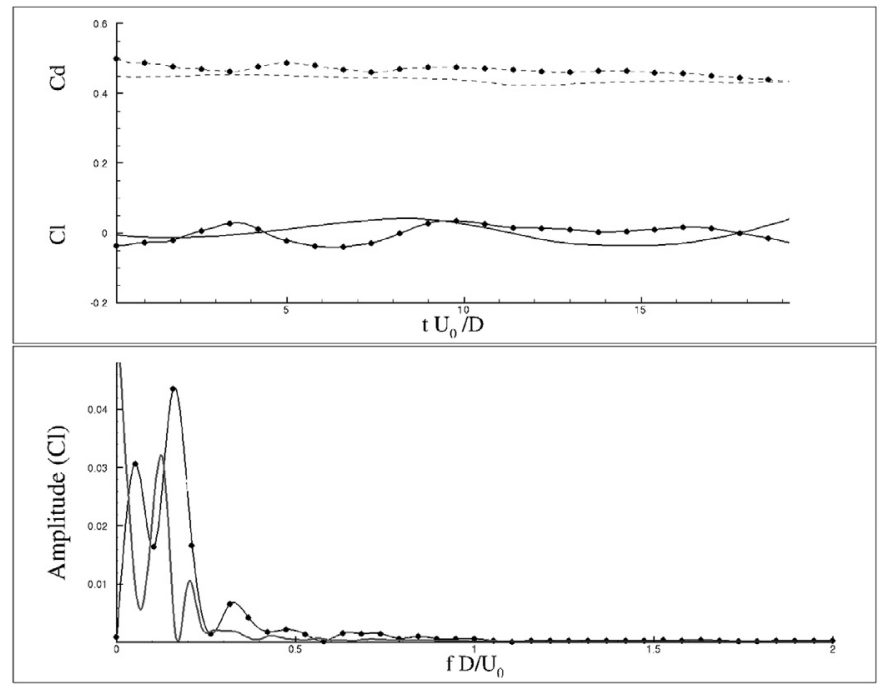

Fig. 4. Time record of the lift coefficient $C l$ and drag coefficient $C d$ for the sphere (top panel). $\mathrm{Cl}$ in the frequency domain (bottom panel). CG, lines; FG, lines with dots.

Although the CG is able to give results in reasonable agreement with the DNS data, the FG reproduces much better the velocity profiles. This is particularly true at the equatorial plane where the FG perfectly reproduces the thin boundary layer developing along the wall-normal direction and at the leading edge $(x=0.5 D)$. In the wake, a small disagreement is present, which may also be attributed to differences in the geometrical configurations. Specifically, we consider an isolated sphere, whereas in the DNS the authors considered a solid stick supporting the sphere at the rear, likewise in laboratory experiments.

The distribution of the skin friction coefficient $C_{\tau}=\left\langle\tau_{w}\right\rangle R e / \rho_{0} U_{0}^{2}$ and of the pressure coefficient $C_{p}=\left(p-p_{0}\right) / 0.5 \rho_{0} U_{0}^{2}$ ( $p_{0}$ is the pressure at the inlet) around the circumferential direction are in Figs. 2 and 3 respectively. These quantities are averaged over time and azimuthal direction. The skin friction coefficient is compared with DNS data of Seidl et al. (1998); the comparison is very good also for this quantity for the FG. Conversely, some disagreements appear for the CG case. This occurs both in the region of attached flow as well as in the separation region, where the change of sign of the shear stress is not reproduced with accuracy. The pressure coefficient is compared with experimental data of Kim and Durbin (1988). In both cases, our LESs reproduce very well the angular distribution of the coefficient as well as of its minimum, well captured at $\theta=71^{\circ}$.

The lift coefficient $C l=F_{y} /\left(0.5 \rho_{0} U^{2} A\right)$ obtained in our simulations exhibits an oscillatory behavior (Fig. 4, top panel). Here $y$ and $z$ are a couple of mutually orthogonal axes contained in a plane orthogonal to the axis $x$ running along the freestream direction (see Fig. 7). The shape of the signal obtained with the FG suggests the presence of a larger number of discrete frequencies. In fact, Fig. 4 (bottom panel) reveals the presence of three main peaks in case of FG. The three peaks correspond to $S t=0.07,0.16$ and 0.32 . The CG is not able to reproduce such dynamics, giving two main peaks only, corresponding to the values of $S t=0.127$ and 0.2 respectively.

The drag coefficient $C_{d}=F_{x} /\left(0.5 \rho_{0} U^{2} A\right)$ also exhibits a weakly oscillatory behavior. The mean value of the drag coefficient is 0.47 for the FG and 0.43 for the CG. Both values are in agreement with literature results (in the range $0.4-0.5$ for Reynolds numbers in the range $10^{3} \div 2 \times 10^{5}$.

To summarize, overall, our LES results exhibit a good agreement with reference DNS and experimental data. As expected, the FG exhibits a better agreement with reference data than the CG. Specifically the FG

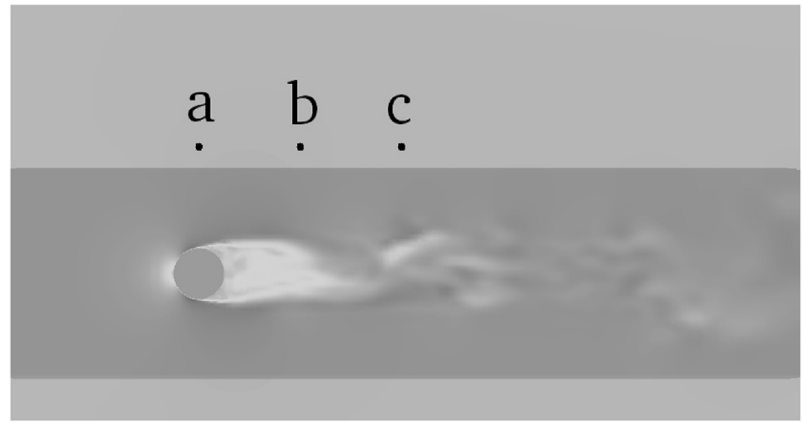

Fig. 5. Validation tests for the acoustic solver. Longitudinal cut (plane $z=0$ ) of the computational domain with a cut of the box (in evidence) used for the volume integration of the quadrupole term: The probes locations are $a(0,2 D, 0), b(2 D, 2 D, 0)$ and $c$ $(4 D, 2 D, 0)$.

is able to reproduce a richer frequency contain of the signal. For this reason, the simulation for the other geometries (cube and prolate spheroid) are carried out on a fine grid, shaped likewise the sphere case.

The acoustic solver has already been validated in Cianferra et al. (2017). Here, for sake of completeness, we repeat some validation tests considering the flow herein investigated. Specifically, we test the acoustic solver by a direct comparison of the pressure signals provided by the FW-H equation with the hydrodynamic pressure provided by LES for three probes with coordinates $(0,2 D, 0),(2 D, 2 D, 0)$ and $(4 D, 2 D, 0)$ respectively. The volume domain considered for solving the quadrupole terms of acoustic equation, that cannot contain the microphone, is sketched in Fig. 5, which shows the section $z=0$ of the computational domain.

Along the z-axis it is $14 D$ long, it covers the entire length along the $x$-axis, and along the $y$-axis the domain is $3 D$ wide. The volume thus extends up to $0.5 D$ from the probes. The results of the validation test are shown in Fig. 6. Specifically we show the spectra level obtained with the two signals. The amplitude is reported in the logarithmic unit decibels (dB). The spectrum level was calculated as $20 \times \log _{10}\left(F F T\left(p^{\prime}\right) /\right.$ $p_{\text {ref }}$ ), where FFT denotes the Fast Fourier Transform of the signal (that was normalized by the number of sample points) and $p_{r e f}=1 \mu \mathrm{Pa}$ is the reference pressure adopted for underwater sound pressure level measurements. On the left panels we report the comparison for the CG, on the right panels we show the results for the FG. The agreement between the signals is very good in both cases, although in case of FG the reconstruction of the signal appears slightly better, in particular in the range of high frequencies. This is in agreement with the findings of Farassat and Brentner (2003) who emphasized the need of fine grids for an accurate reconstruction of the signal.

Hereafter, when referring to LES data for the sphere, we implicitly make reference to those obtained with the fine grid.

Note that the frequency-dependent hearing threshold of human ears in underwater conditions is relatively high, $84-100 \mathrm{~dB}$, when referenced to $1 \mu \mathrm{Pa}$, and the ambient noise is in the range of $60-100 \mathrm{~dB}$.

\subsection{Comparative analysis for the three bodies}

First, we show instantaneous streamtracers for the three bodies investigated, to give a qualitative sight of the flow at the rear of the three objects (Fig. 7). Flow separation is substantially different in the three cases: The separation angle on the sphere is about $90^{\circ}$; the main recirculation beyond the sphere covers a long region up to $x=2 D$ where the mean flow reattaches, while smaller recirculation spots are present in the proximity of the surface. The snapshot captures a large vortex released at the top side of the sphere and a number of small structures close to the body surface, result of the small-scale shear layer 

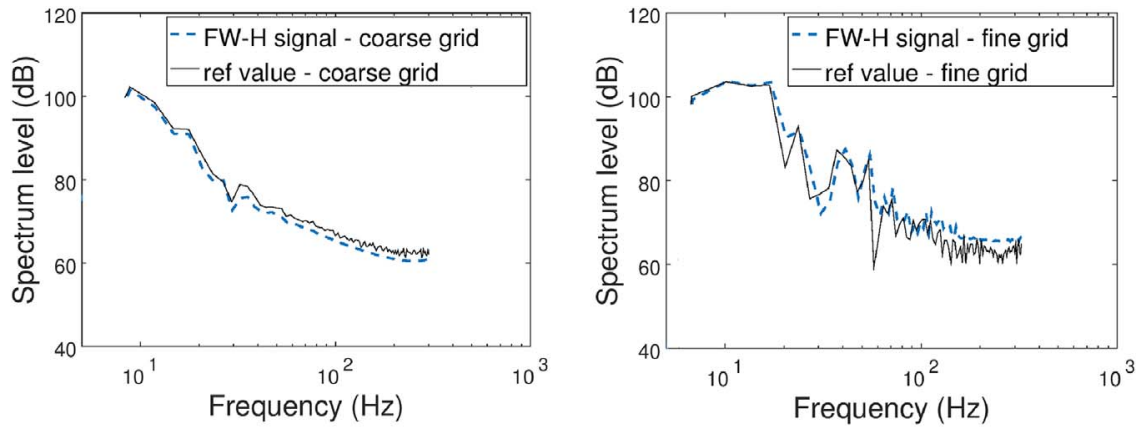

Fig. 6. Validation tests for the acoustic solver. Sound spectrum level predicted by LES (solid line) and by the FW-H equation (dashed line) at probes: A $(0,2 D, 0)$, top panel; b $(2 D, 2 D, 0)$, center panel; c, $(4 D, 2 D, 0)$ bottom panel.
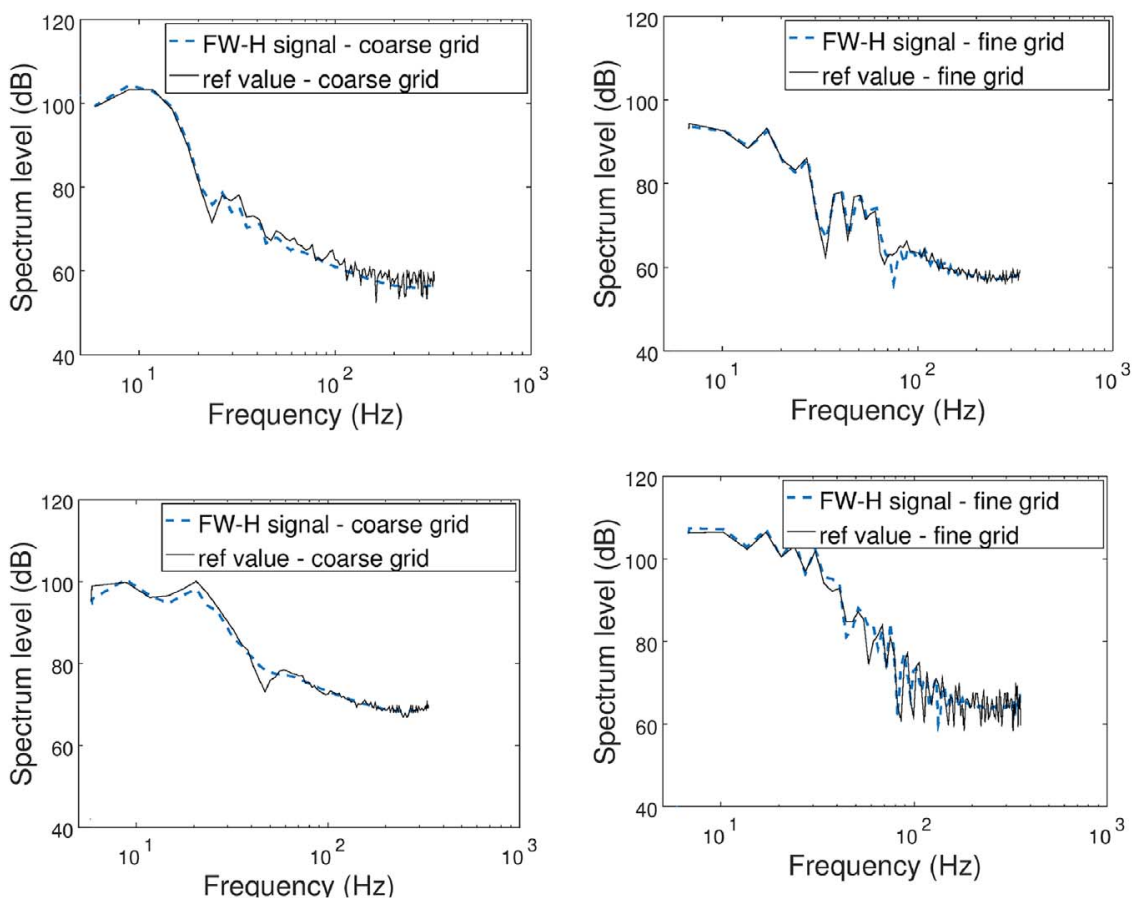

developing beyond separation. The flow over the cube separates at the front corners causing a widening of the mean flow which tends to reattach about $1 D$ in the downstream region. Small-scale vorticity is appreciable over the freestream-oriented surfaces. Finally, the streamlined profile of the prolate spheroid gives rise to a recirculation region in the very rear part of the body, small compared to that of the previous cases. The main vortex behind the spheroid (depicted in Fig. 7) has a diameter of about $0.28 D$ compared to those behind the sphere $(\sim 1 D)$ and behind the cube $(\sim 0.8 D)$. The flow over the prolate spheroid separates at $x=2.7 D$, at the ending part of the body.

In order to get a qualitative view of the turbulent wake, contour plots of the resolved turbulent kinetic energy $k=\frac{1}{2}\left(\left\langle u^{\prime} u^{\prime}\right\rangle+\left\langle v^{\prime} v^{\prime}\right\rangle+\left\langle w^{\prime} w^{\prime}\right\rangle\right)$ (the symbol ' denotes resolved fluctuations) are depicted in Fig. 8 . The time window over which the quantity was calculated is not enough to obtain convergent second-order statistics, however, for the scopes of the present paper, the figure gives useful information. Three planes, orthogonal to the flow direction, were selected for this analysis, located respectively at distances $D, 3 D$ and $6 D$ respectively, from the trailing edge of the bodies.

For a certain body, the region of high $k$ intensifies and increases in size moving downstream (panels from the left to the right). At a certain distance from the trailing edge, for the three bodies (panels from the top to the bottom) we observe that the cube produces the highest level of $k$ in a wider region. The intensity of the wake behind the sphere is comparable to that of the cube although a bit smaller in size. The prolate spheroid produces small levels of $k$ over a small region (note the different spatial scales of the bottom panels with respect to the others in Fig. 8). These differences are expected to affect the acoustic signals.

In order to study the acoustic far-field we analyzed the pressure signals reconstructed by the FW-H equation at 6 different probes, whose coordinates are in Table 1 . Two probes are over the longitudinal axis $x$ in the far wake $\left(A_{0}\right.$ and $\left.A_{1}\right)$; two over the transversal axis $y$ in the far field $\left(C_{0}\right.$ and $\left.C_{1}\right)$ and two over a diagonal oriented at 45 degrees in the $x-y$ plane $\left(B_{0}\right.$ and $\left.B_{1}\right)$, in the far field. The domain considered to compute the quadrupole terms of the FW-H equation is the volume centered over the body and enclosed in a box with dimension $12 D \times 12 D$ in the $y, z$ directions, and running along the entire computational domain along the $x$-axis.

The time-signals of the bluff bodies turn out to be the most intense and richest in frequencies (Fig. 9). The sphere and cube produce equivalent far-field noise. The noise radiated by the prolate spheroid is substantially smaller than that of the other bodies. 

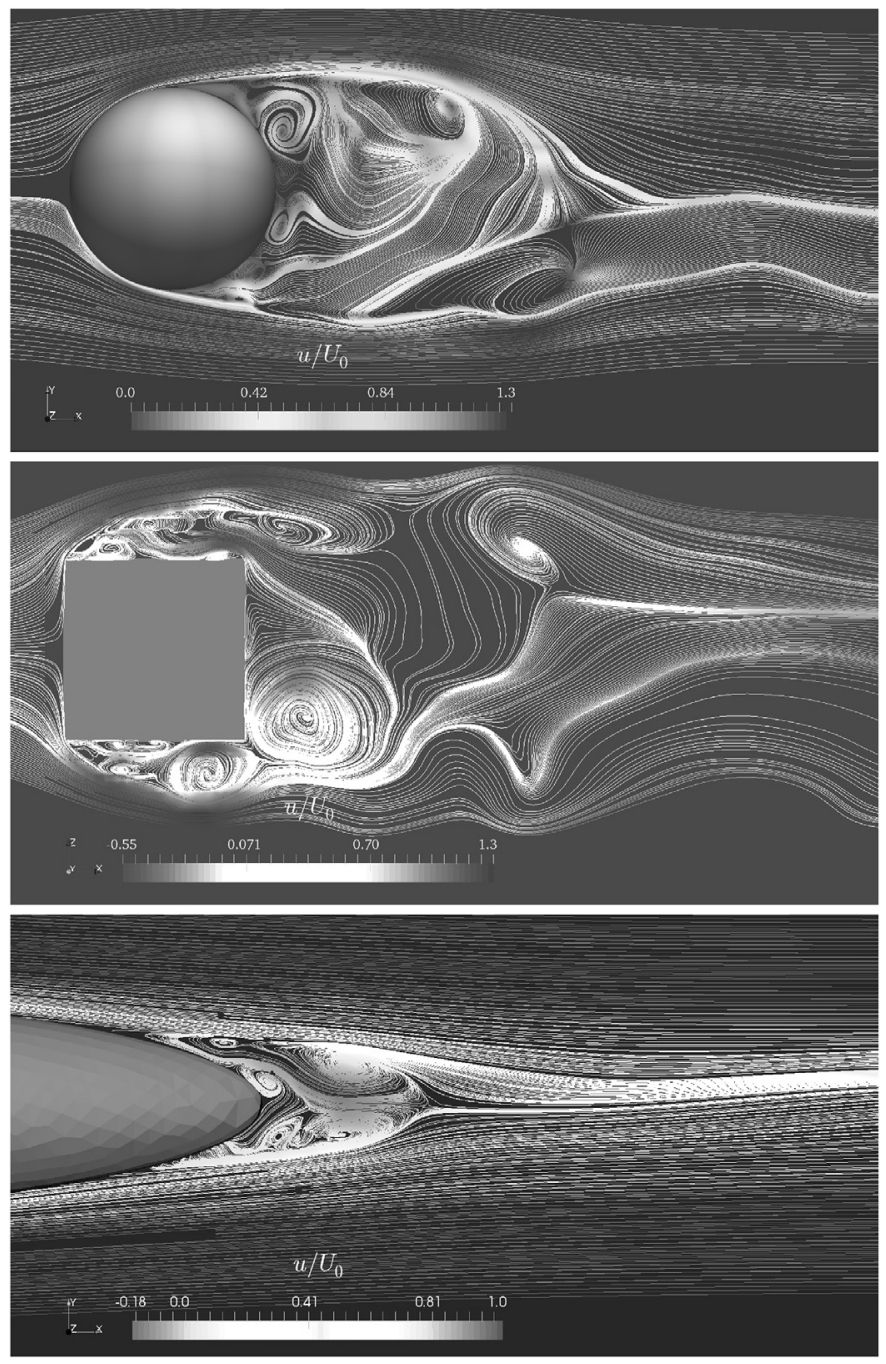

Fig. 7. Snapshots of the flow around sphere (top panel), cube (middle panel) and prolate spheroid (bottom panel). Instantaneous streamtracers together with contour of the streamwise velocity, made non-dimensional with the inflow velocity $U_{0}$.

As well known, the FW-H equation consists of a sum of linear terms, related to the integration on the body surface (loading noise), and nonlinear terms, corresponding to a volume integration on the flowsource region. Thus, it may be interesting to evaluate the separate contributions coming from the different terms.

We show the spectrum level from the linear source terms in Fig. 10 and from the nonlinear sources in Fig. 11 respectively. Overall, the analysis of Fig. 10 suggests that the loading noise deriving from the bluff bodies is substantially larger than that coming from the streamlined body, thus confirming that its elongated, hydrodynamic shape produces a very low (loading) noise. This occurs at all probe positions investigated. Further, the energy content in the high frequencies appears a bit larger in the case of the cube than for the sphere. This may be the effect of the small scale vorticity developing because of the sharp corners. In fact, the high frequencies appear more the linear part of the signal, while nonlinear terms seem to provide mainly low frequencies.

The sound spectrum level of the non-linear part of the signal (Fig. 11) gives information on the sound level mostly generated by the wakes developing downstream. The loudness of the wakes appear comparable among the three cases downstream the bodies in the far wake (microphones $A_{0}$ and $A_{1}$ ). Conversely, at noticeable distance from the bodies, along the transversal directions (microphones $B_{1}$ to $C_{2}$ ), the wakes generated by the bluff bodies appear more loudly than that of the prolate spheroid. This suggests a sort of directivity of the noise generated by the hydrodynamically streamlined body, compared to the two cases of bluff bodies.

Finally, we observe that, for bluff bodies, the linear contribution to the far field noise is always more significant than its non-linear counterpart. The opposite is true for the prolate spheroid, due to the very weak loading noise generated by this body. In general, this behavior may be explained by referring to the FW-H equation, where, as well known, the far field components of linear terms decay as $1 / r$ ( $r$ being the source-observer distance), while the decay of the nonlinear source contributions is notoriously much faster.

\subsection{Cube versus square cylinder}

This section is devoted to a preliminary comparison between the hydroacoustic field generated by the cube, as presented in the previous section, and by an elongated cylinder with a squared section, studied in Cianferra et al. (2017). In that paper, a cylinder was immersed in a uniform flow at a Reynolds number equal to 4000 (based on the section side $d$ ) while the length of the body was equal to $30 d$. The "aspect ratio" $A R$ of the body (defined as the ratio between the cylinder length and the side of the squared section) was 30 , while in the case of a cube $A R=1$. Both fluid-dynamics and acoustics data were obtained using the same methodologies. In the case of cube the domain size was long enough to allow destruction and dissipation of the coherent part of the wake. In case of cylinder, the elongated shape of the body suggested adoption of a very elongated mesh $(100 d \times 70 d \times 80 d)$, in order to provide an accurate reproduction of the well known von Karman coherent wake.

It has to be pointed out that in Cianferra et al. (2017) the acoustic analysis of the pressure signals was carried out considering air as a medium. Also, the inlet velocity as well as the dimension of the body were different from those of the cube discusses in the previous Section. Consequently, for making the present comparison consistent, first we re-processed the pressure signal relative to the cylinder considering water as the carrying fluid. Then we present the results in non-dimensional form, thus showing the spectrum of the pressure made non dimensional with $\rho_{0} U_{0}^{2}$ as a function of the non-dimensional frequency $f d$ / $U_{0}$.

Fig. 12 shows a comparison between velocity fields determined in fully developed conditions for the two cases. Specifically, the comparison is shown on the longitudinal $x-y$ mid-plane of the two computational domains. As expected, in the mid-section the flow downstream the cylinder is characterized by the presence of a persisting and oscillating wake, which becomes progressively wider and finally breaks, turning into a rather chaotic and turbulent flow. On the other hand, the wake behind the cube is more irregular and, above all, spreads out much earlier in the field, thus providing a much weaker occurrence of nonlinear noise sources in a region limited in space.

Fig. 13 shows a comparison between the noise non-dimensional spectra at probe $A 1$ (located $100 D$ downstream of the body), corresponding to both linear (left panel) and nonlinear (right panel) FW-H source terms. Apart the extent of the spectra in the frequency domain, related, from one side, to the extension of the available time window and, from the other side, to the sampling time interval, the figures give very useful information. The loading terms given by the two bodies overlap in the regions of non-dimensional frequencies in common. This is not surprising, since the loading term is mainly related to the shape of 


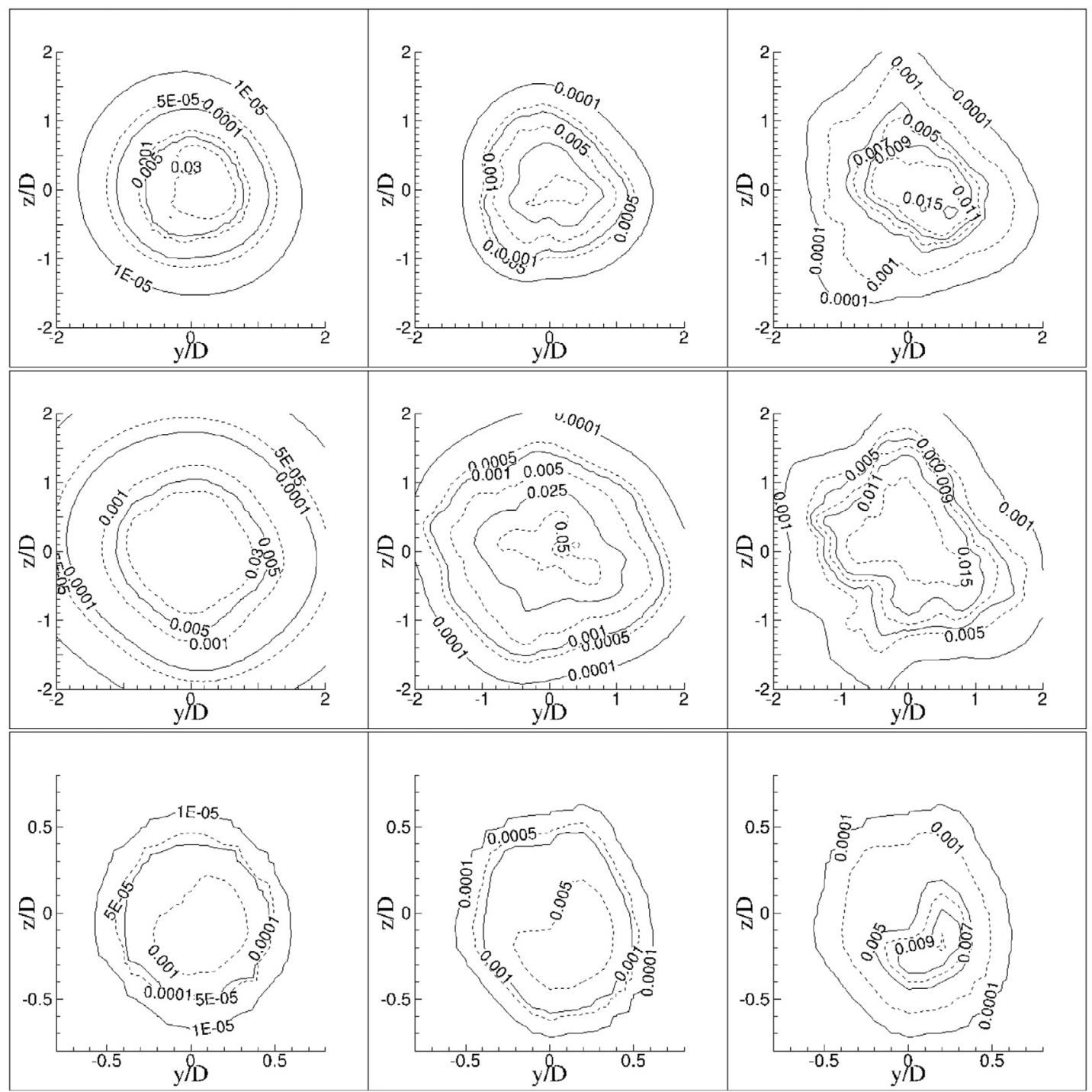

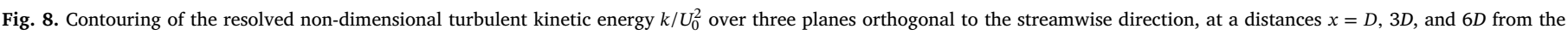
trailing edge of the bodies (from left to right panels): Sphere (top panels); cube (middle panels); prolate spheroid (bottom panels).

Table 1

Coordinates of probes at $z=0$ placed in the far field for the analysis of the radiated noise.

\begin{tabular}{|c|c|c|c|c|c|c|}
\hline & A0 & $\mathrm{A} 1$ & B0 & B1 & $\mathrm{CO}$ & $\mathrm{C} 1$ \\
\hline$x$ & $50 D$ & $100 D$ & $50 D$ & $100 D$ & 0 & 0 \\
\hline$y$ & 0 & 0 & $50 D$ & $100 D$ & $50 D$ & $100 D$ \\
\hline
\end{tabular}

the cross-sectional section that, in the two cases investigated is the same (a square). On the other side, substantial differences are observed in the non-dimensional spectra of the non-linear quadrupole terms, the signal given by the wake of the cylinder being much larger than that given by the cube. This has to be attributed to the energy content of the 2Dshaped wake developing behind the cylinder, when compared to the 3D-shaped wake developing around the cube. This important aspect will be exploited in a successive work.

\section{Conclusions}

In the present paper we analyzed the far-field noise radiated by three archetypal, three-dimensional, bodies placed in a uniform current. We studied a sphere, a cube and a prolate spheroid at a value of the Reynolds number, based on the square root of the frontal area, $R_{A}=4430$. We used the acoustic analogy, solving the turbulent fluid dynamic field through wall-resolving LES and the FW-H equation for the evaluation of the acoustic far-field noise. The study has allowed generation of a fluid-dynamic LES database for three significant geometries.

The quadrupole term of the FW-H equation was first re-formulated in the convective form to deal with a problem characterized by the presence of a uniform current and, then, solved through direct volume integration. This procedure, unaffordable in presence of time delays rising when the noise source is not compact, here as well as in 

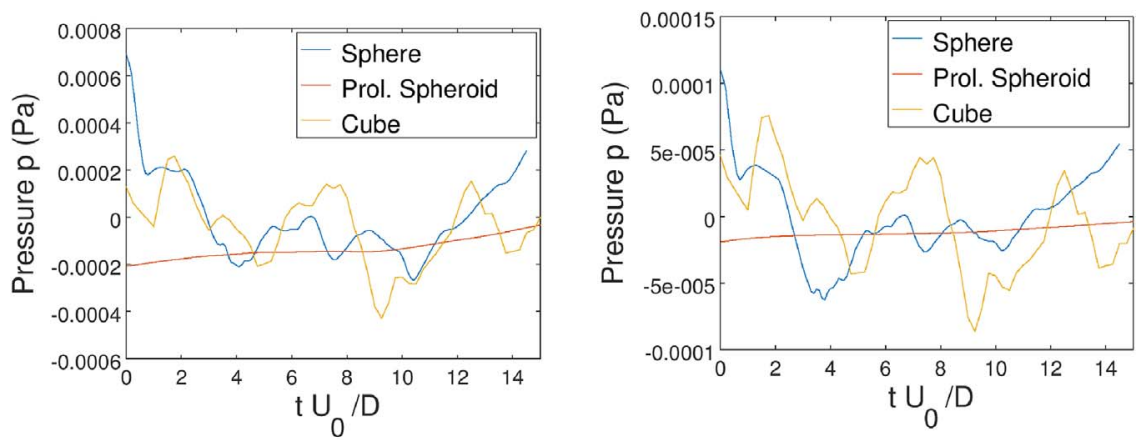

Fig. 9. Pressure signal reconstructed by the $\mathrm{FW}-\mathrm{H}$ equation at 6 different probes $A 0, \ldots, C 1$, their coordinates are in Table 1.
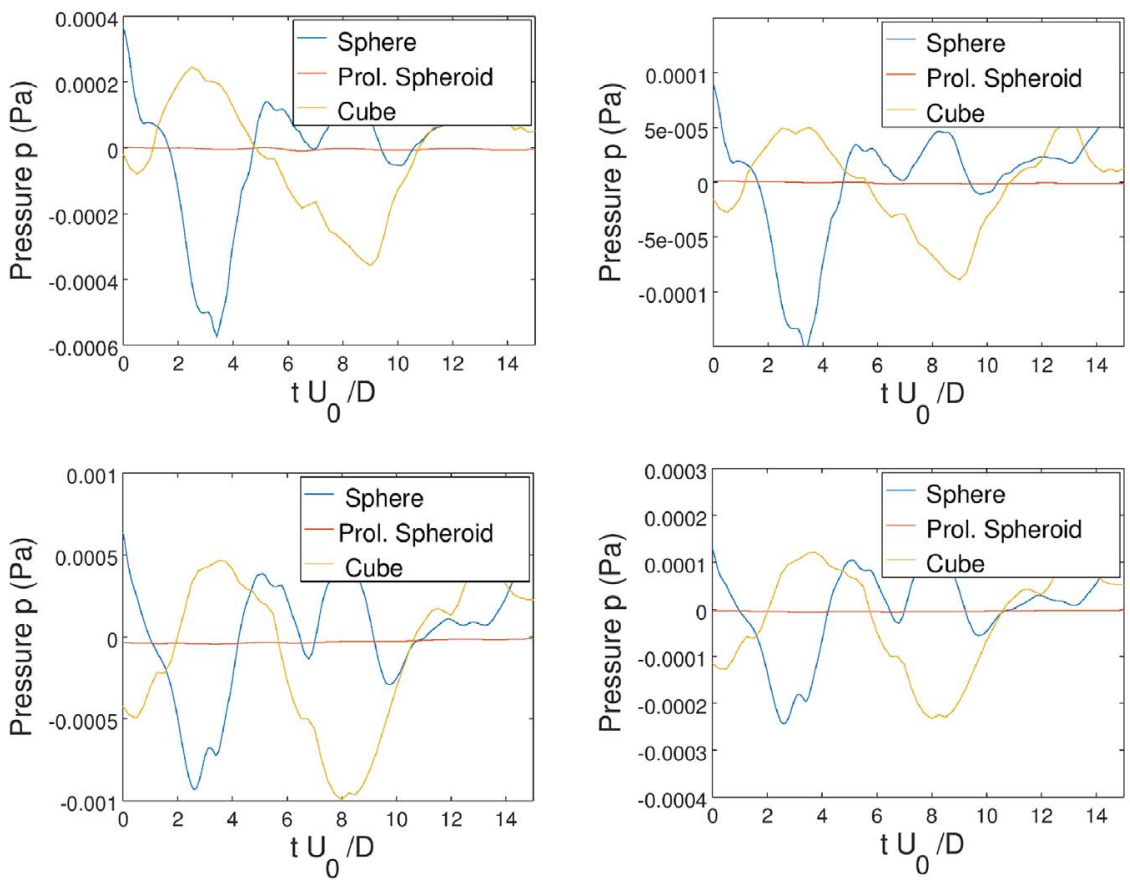

literature, was shown to be applicable in hydrodynamics, where the speed of sound is very high and the flow velocity is small. Validation tests were performed to show the accuracy of the methodology.

The analysis of the fluid-dynamic data showed that the cube has the widest and most energetic and irregular wake mainly associated to the sharp corners. At the opposite stands the prolate spheroid, whose the streamlined shape provides a small slender wake characterized by a low level of turbulent kinetic energy. These differences were shown to impact the generated hydrodynamic noise.

The comparison of the acoustic field generated by sphere, cube and ellipsoid demonstrate how body shape is decisive in the generation of different types of hydroacoustic noise. Specifically, the two bluff bodies appear to be the loudest with a signal composed of a wide spectrum of frequencies; the prolate spheroid provides a pressure signal one order of magnitude lower than the other cases, associated to a very low frequency.

The analysis of the contribution of the different terms of the FW-H equation showed that for the bluff bodies (cube and sphere) the linear terms contribute to the overall noise signal more than the non-linear ones. The opposite is true for the streamlined body (prolate spheroid), since its own shape makes the loading noise very small.

Finally, a preliminary comparison between the noise generated by the cube and by a square elongated cylinder, shows that the aspect ratio of the body might rule the relative importance between linear and nonlinear contributions to the acoustic field as well as the level of noise generated by the wake. This has been attributed to the persistence of the two-dimensionally shaped wake in case of elongated cylinder when compared to the three-dimensional one generated by the cube. This issue deserves a successive study. 

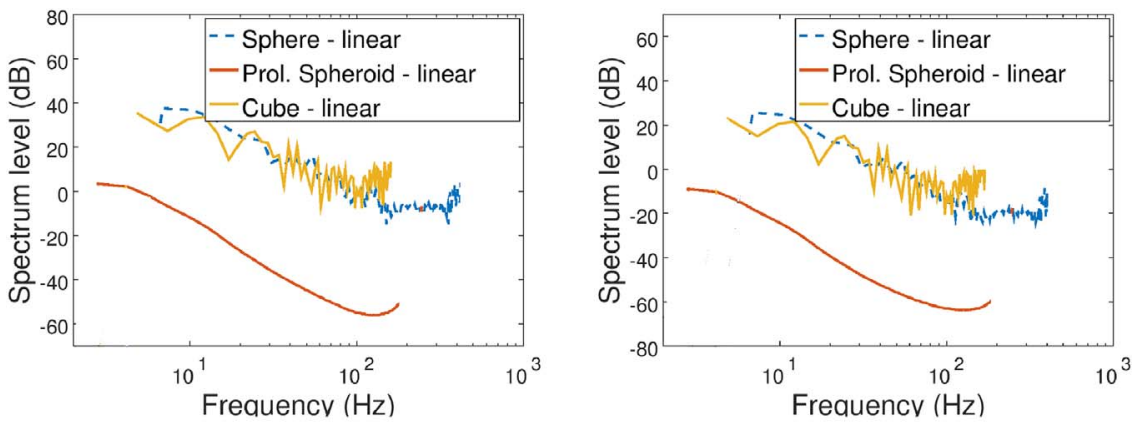

Fig. 10. Sound spectrum level of the linear terms of FW-H in at all probes probes of Table 1 .
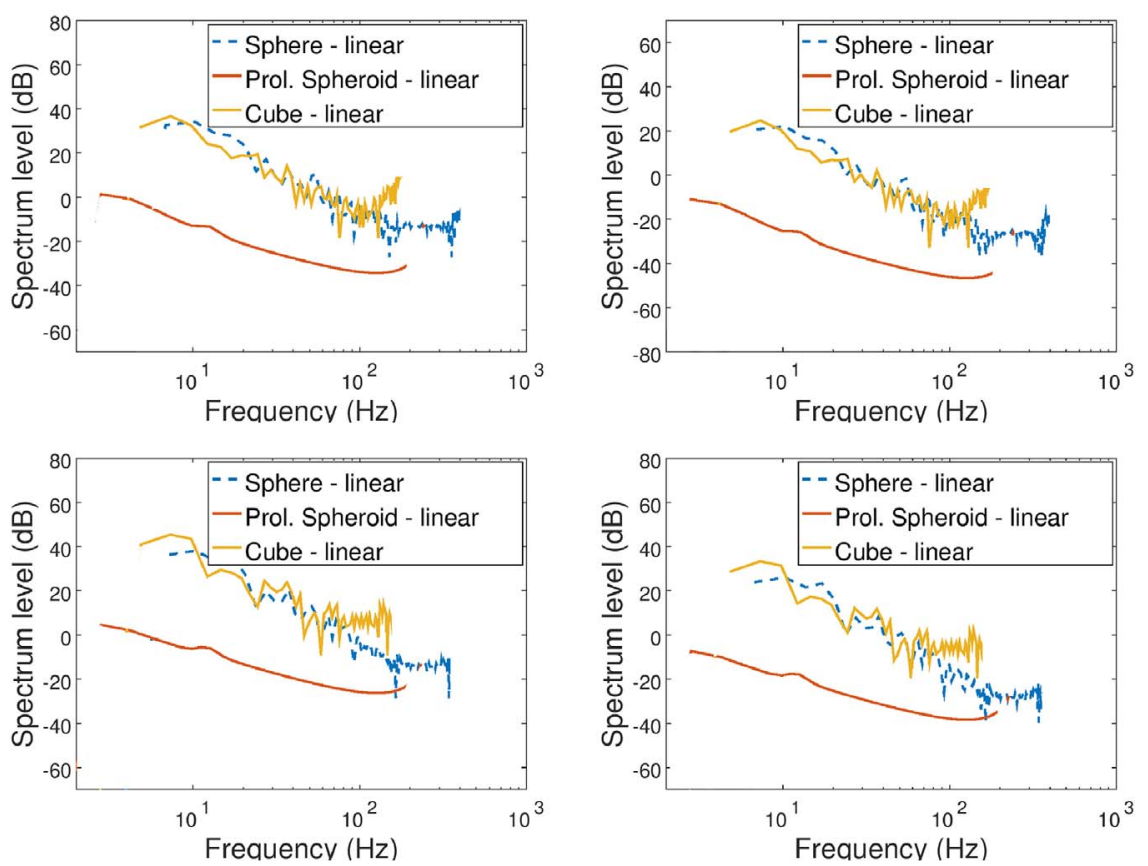

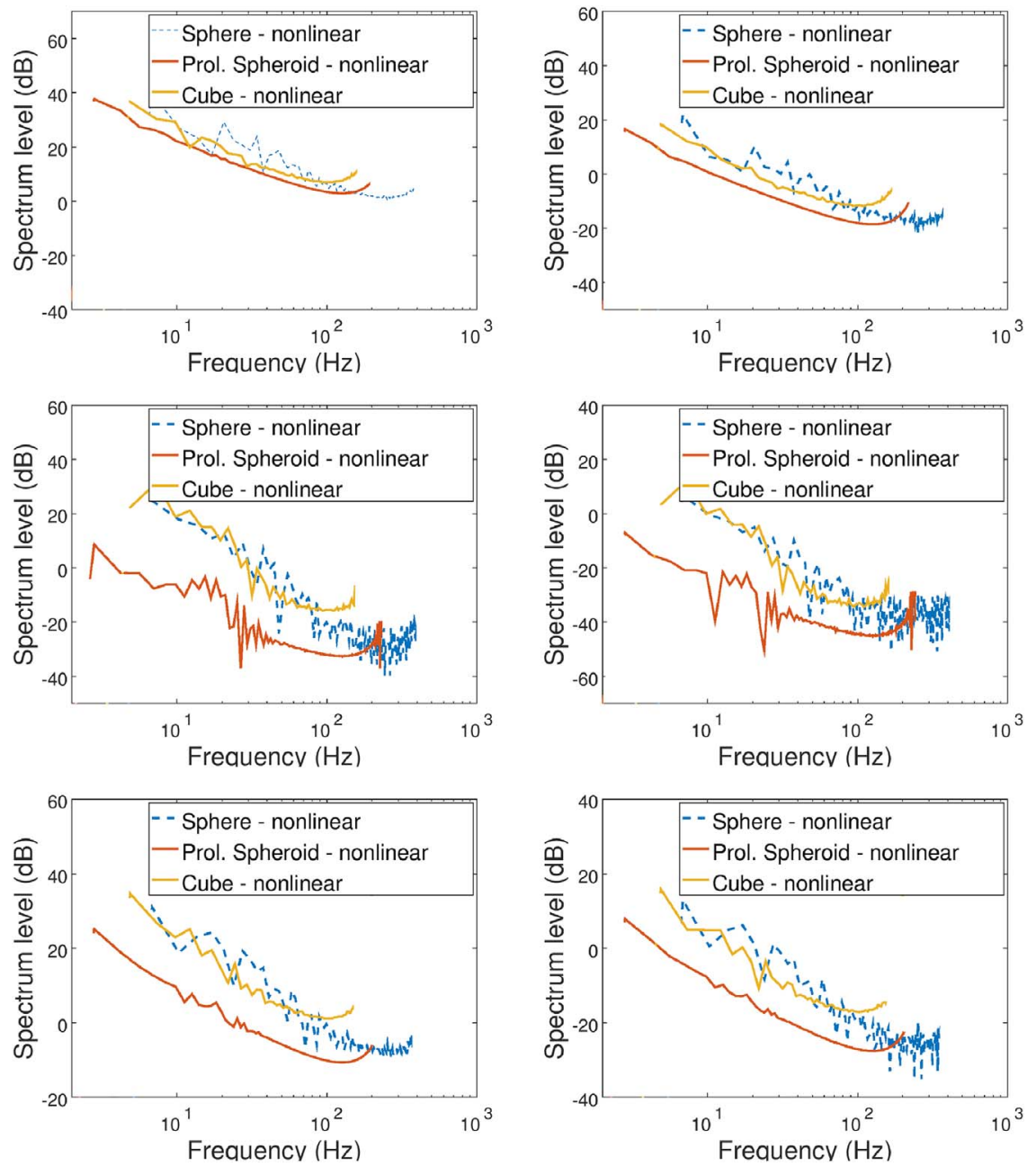

Fig. 11. Sound spectrum level of the non-linear terms of FW-H in at all probes probes of Table 1 .

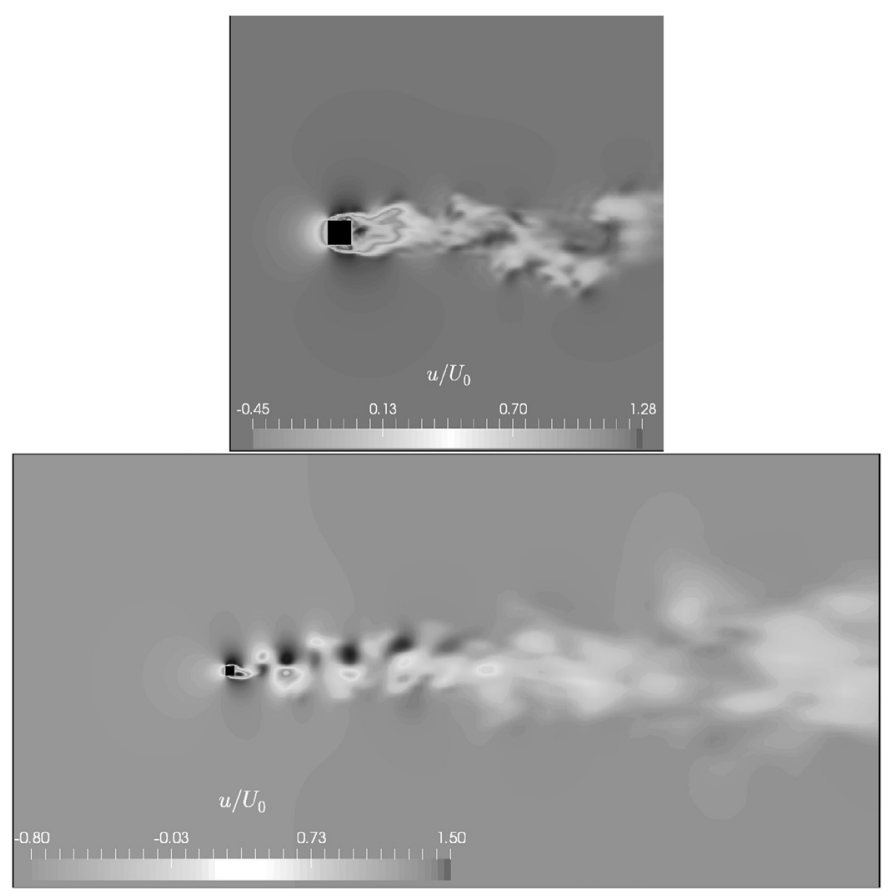

Fig. 12. Comparison of instantaneous streamline velocity $u$, made non-dimensional by the inlet uniform velocity $U_{0}$, in the case of a flow around a cube (top panel) and flow around a square cylinder (bottom panel). 


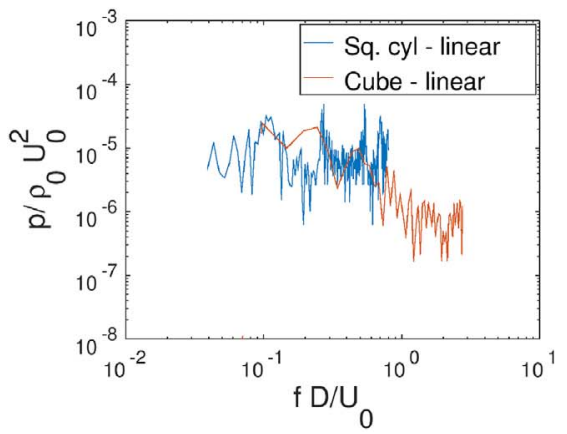

Acknowledgments

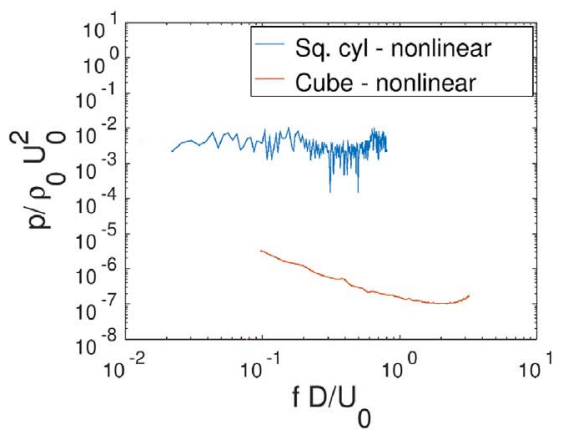

2017-2020), Regione Friuli Venezia Giulia, Italy.

The present research has been supported by PRELICA (POR-FESR

\section{Appendix A}

In this section we report some details about the mathematical manipulation of the convective FW-H equation and, in particular, the procedure which allows to write down the integral form of the nonlinear terms (4). The convective FW-H equation may be easily obtained from the conservation laws of mass and momentum, by accounting for the presence of a medium moving at a constant velocity $\mathbf{U}_{0}$; then, at each point, the fluid velocity is $\mathbf{U}_{0}+\mathbf{u}$, being $\mathbf{u}$ the local perturbation velocity, and the equation reads Najafi-Yazdi et al. (2011)

$\square_{C}^{2} p^{\prime}(\mathbf{x}, t)=\left(\frac{\partial}{\partial t}+U_{0 j} \frac{\partial}{\partial x_{j}}\right)\left[Q_{k} \hat{n}_{k} \delta(f)\right]-\frac{\partial}{\partial x_{i}}\left[L_{i j} \hat{n}_{j} \delta(f)\right]+\frac{\partial^{2}}{\partial x_{i} \partial x_{j}}\left[T_{i j} H(f)\right]$

where all derivatives have to be accounted for in a "generalized" sense, $\delta(f)$ and $H(f)$ are the Dirac and Heaviside functions, respectively, and

$\square_{C}^{2}=\left[\frac{\partial^{2}}{\partial t^{2}}-c_{0}^{2} \frac{\partial^{2}}{\partial x_{i} \partial x_{j}}+2 U_{0 j} \frac{\partial^{2}}{\partial t \partial x_{j}}+U_{0 i} U_{0 j} \frac{\partial^{2}}{\partial x_{i} \partial x_{j}}\right]$

represents the convective form of the D'Alembert operator. Furthermore

$Q_{k}=\left[\rho\left(u_{k}+U_{0 k}-v_{k}\right)+\rho_{0}\left(v_{k}-U_{0 k}\right)\right]$

$L_{i j}=\left[\rho u_{i}\left(u_{j}+U_{0 j}-v_{j}\right)+P_{i j}\right]$

$T_{i j}=\left[\rho u_{i} u_{j}+P_{i j}-c_{0}^{2} \tilde{\rho} \delta_{i j}\right]$

where $P_{i j}$ is the compressive stress tensor, $\widetilde{\rho}$ the perturbation density, $c_{0}$ the sound speed and $\delta_{i j}$ the Kronecker delta. Without loss of generality, it can be assumed that the mean flow velocity $\mathbf{U}_{0}$ is along the positive $x_{1}$-direction. By using the convective form of the free-space Green function

$G(\mathbf{x}, t ; \mathbf{y}, \tau)=\frac{\delta(g)}{4 \pi r^{*}} \quad$ with $\quad g=\tau-t+\frac{r}{c_{0}}$,

where $(\mathbf{x}, t)$ and $(\mathbf{y}, \tau)$ are the observer and source position and time, respectively, and

$$
\begin{aligned}
& r^{*}=\sqrt{\left(x_{1}-y_{1}\right)^{2}+\beta^{2}\left[\left(x_{2}-y_{2}\right)^{2}+\left(x_{3}-y_{3}\right)^{2}\right]} ; \\
& r=\frac{-M_{0}\left(x_{1}-y_{1}\right)+r^{*}}{\beta^{2}} ; \quad \beta=\sqrt{1-M_{0}^{2}} ;
\end{aligned}
$$

with the components of the unit radiant vectors given by

$\hat{r}_{1}^{*}=\frac{\left(x_{1}-y_{1}\right)}{r^{*}} \quad ; \quad \hat{r}_{2}^{*}=\beta^{2} \frac{\left(x_{2}-y_{2}\right)}{r^{*}} ; \quad \hat{r}_{3}^{*}=\beta^{2} \frac{\left(x_{3}-y_{3}\right)}{r^{*}}$

and

$\hat{r}_{1}=-\frac{M_{0}}{\beta^{2}}+\frac{1}{\beta^{2}} \frac{\left(x_{1}-y_{1}\right)}{r^{*}} \quad ; \quad \hat{r}_{2}=\frac{\left(x_{2}-y_{2}\right)}{r^{*}} ; \quad \hat{r}_{3}=\frac{\left(x_{3}-y_{3}\right)}{r^{*}}$,

Eq. (5) may be turned into an integral form. The first two source terms on the right-hand-side give rise to the surface integrals of Eq. (3)and the procedure is described in detail in Najafi-Yazdi et al. (2011). An analogous transformation may be carried out on the third (quadrupole) term, which, in essence, requires the manipulation of the double spatial derivative of the Green function

$\frac{\partial^{2}}{\partial x_{i} \partial x_{j}}\left[\frac{\delta(g)}{r^{*}}\right]$

Starting from

$\frac{\partial}{\partial x_{i}}\left[\frac{\delta(g)}{r^{*}}\right]=-\frac{\hat{r}_{i}}{c_{0} r^{*}} \frac{\partial}{\partial t}[\delta(g)]-\frac{\hat{r}_{i}^{*}}{r^{* 2}} \delta(g)$ 
we have

$$
\begin{aligned}
\frac{\partial}{\partial x_{j}}\left\{\frac{\partial}{\partial x_{i}}\left[\frac{\delta(g)}{r^{*}}\right]\right\} & =\frac{\partial}{\partial x_{j}}\left\{-\frac{1}{c_{0}} \frac{\hat{r}_{i}}{r^{*}} \frac{\partial}{\partial t}[\delta(g)]-\frac{\hat{r}_{i}^{*}}{r^{* 2}} \delta(g)\right\} \\
& =-\frac{1}{c_{0}} \frac{\partial}{\partial x_{j}}\left[\frac{\hat{r}_{i}}{r^{*}}\right] \cdot\left\{\frac{\partial}{\partial t}[\delta(g)]\right\}-\frac{1}{c_{0}} \frac{\hat{r}_{i}}{r^{*}} \cdot\left\{\frac{\partial}{\partial x_{j}}\left(\frac{\partial}{\partial t}[\delta(g)]\right)\right\}-\frac{\partial}{\partial x_{j}}\left[\frac{\hat{r}_{i}^{*}}{r^{* 2}}\right] \cdot\{\delta(g)\}-\frac{\hat{r}_{i}^{*}}{r^{* 2}} \cdot\left\{\frac{\partial}{\partial x_{j}}[\delta(g)]\right\}
\end{aligned}
$$

From the relations

$\frac{\partial}{\partial x_{j}}\left[\frac{\hat{r}_{i}}{r^{*}}\right]=-\frac{1}{r^{*}} \frac{\partial \hat{r}_{i}}{\partial x_{j}}-\frac{\hat{r}_{i} \hat{r}_{j}^{*}}{r^{* 2}}$

$\frac{\partial}{\partial x_{j}}\left(\frac{\partial}{\partial t}[\delta(g)]\right)=-\frac{\hat{r}_{j}}{c_{0}} \frac{\partial^{2}}{\partial t^{2}}[\delta(g)]$

$\frac{\partial}{\partial x_{j}}\left[\frac{\hat{r}_{i}^{*}}{r^{* 2}}\right]=\frac{1}{r^{* 2}} \frac{\partial \hat{r}_{i}^{*}}{\partial x_{j}}-2 \frac{\hat{r}_{i}^{*} \hat{r}_{j}^{*}}{r^{* 3}}$

$\frac{\partial}{\partial x_{j}}[\delta(g)]=-\frac{\hat{r}_{j}}{c_{0}} \frac{\partial}{\partial t}[\delta(g)]$

we obtain

$\frac{\partial^{2}}{\partial x_{i} \partial x_{j}}\left[\frac{\delta(g)}{r^{*}}\right]=+\frac{1}{c_{0}^{2}} \frac{\hat{r}_{i} \hat{r}_{j}}{r^{*}} \frac{\partial^{2}}{\partial t^{2}}[\delta(g)]+\frac{1}{c_{0}}\left[\frac{2 \hat{r}_{i} \hat{r}_{j}^{*}}{r^{* 2}}-\frac{1}{r^{*}} \frac{\partial \hat{r}_{i}}{\partial x_{j}}\right] \frac{\partial}{\partial t}[\delta(g)]+\left[\frac{2 \hat{r}_{i}^{*} \hat{r}_{j}^{*}}{r^{* 3}}-\frac{1}{r^{* 2}} \frac{\partial \hat{r}_{i}^{*}}{\partial x_{j}}\right] \delta(g)$

Concerning the spatial derivative of $\hat{r}_{i}^{*}$, we have

$$
\begin{aligned}
\frac{\partial \hat{r}_{i}^{*}}{\partial x_{j}} & =\frac{\partial}{\partial x_{j}}\left[\frac{r_{i}^{*}}{r^{*}}\right]=\frac{1}{r^{*}} \frac{\partial r_{i}^{*}}{\partial x_{j}}-\frac{r_{i}^{*}}{r^{* 2}} \frac{\partial r^{*}}{\partial x_{j}}=\frac{1}{r^{*}} \frac{\partial r_{i}^{*}}{\partial x_{j}}-\frac{\hat{r}_{i}^{*} \hat{r}_{j}^{*}}{r^{*}} \\
& =\frac{1}{r^{*}}\left[\begin{array}{ccc}
1 & 0 & 0 \\
0 & \beta^{2} & 0 \\
0 & 0 & \beta^{2}
\end{array}\right]-\frac{\hat{r}_{i}^{*} \hat{r}_{j}^{*}}{r^{*}}=\frac{1}{r^{*}}\left[R_{i j}^{*}-\hat{r}_{i}^{*} \hat{r}_{j}^{*}\right]
\end{aligned}
$$

where we have set

$R_{i j}^{*}=\left[\begin{array}{ccc}1 & 0 & 0 \\ 0 & \beta^{2} & 0 \\ 0 & 0 & \beta^{2}\end{array}\right]$

On the other hand, by reminding (6) and (7), it is easy to see

$\hat{r}_{1}=-\frac{M_{0}}{\beta^{2}}+\frac{1}{\beta^{2}} \hat{r}_{1}^{*} ; \quad \hat{r}_{2}=\frac{1}{\beta^{2}} \hat{r}_{2}^{*} ; \quad \hat{r}_{3}=\frac{1}{\beta^{2}} \hat{r}_{3}^{*}$

so that

$\frac{\partial \hat{r}}{\partial x_{j}}=\frac{1}{\beta^{2}} \frac{\partial \hat{r}^{*}}{\partial x_{j}}=\frac{1}{\beta^{2} r^{*}}\left[R_{i j}^{*}-\hat{r}_{i}^{*} \hat{r}_{j}^{*}\right]$

Finally, Eq. (8) reads

$\frac{\partial^{2}}{\partial x_{i} \partial x_{j}}\left[\frac{\delta(g)}{r^{*}}\right]=+\frac{1}{c_{0}^{2}} \frac{\hat{r}_{i} \hat{r}_{j}}{r^{*}} \frac{\partial^{2}}{\partial t^{2}}[\delta(g)]+\frac{1}{c_{0}}\left[\frac{2 \hat{r}_{i} \hat{r}_{j}^{*}}{r^{* 2}}+\frac{1}{\beta^{2} r^{* 2}}\left(\hat{r}_{i}^{*} \hat{r}_{j}^{*}-R_{i j}^{*}\right)\right] \frac{\partial}{\partial t}[\delta(g)]+\left[\frac{3 \hat{r}_{i}^{*} \hat{r}_{j}^{*}-R_{i j}^{*}}{r^{* 3}}\right] \delta(g)$

It 's worth pointing out that under the assumption $M_{0} \ll 1$ we have $\beta \approx 1$ and, consequently, $r=r^{*}, R_{i j}^{*}=\delta_{i j}$; then, Eq. (10) reduces to the classic form

$\frac{\partial^{2}}{\partial x_{i} \partial x_{j}}\left[\frac{\delta(g)}{r}\right]=+\frac{1}{c_{0}^{2}} \frac{\hat{r}_{i} \hat{r}_{j}}{r} \frac{\partial^{2}}{\partial t^{2}}[\delta(g)]+\frac{1}{c_{0}}\left[\frac{3 \hat{r}_{i} \hat{r}_{j}-\delta_{i j}}{r^{2}}\right] \frac{\partial}{\partial t}[\delta(g)]+\left[\frac{3 \hat{r}_{i} \hat{r}_{j}-\delta_{i j}}{r^{3}}\right] \delta(g)$

The product of Eq. (10) by the Lighthill tensor $T_{i j}$ exactly gives rise to the integral kernels of Eq. (4).

\section{References}

Achenbach, E., 1974. Vortex shedding from spheres. J. Fluid Mech. 62, 209-221. Bakic, V., 2002. Experimental investigation of turbulent flows around a sphere, Ph.D. Thesis. TUHH Hamburg, Germany.

Bensow, R., Liefvendahl, M., 2016. An acoustic analogy and scale-resolving flow simu-

lation methodology for the prediction of propeller radiated noise. 31st Symposium on Naval Hydrodynamics.

Blokhintsev, D., 1956. Acoustics of a nonhomogeneous moving media. NACA TM-1399.
Carlton, J., Vlasic, D., 2005. Ship vibration and noise: some topical aspects. 1st International Ship Noise and Vibration Conference. pp. 1-11. http://dx.doi.org/10. 1063/1.4930815.

Chomaz, J., Bonnetin, P., Hopfinger, E., 1993. The structure of the near wake of a sphere moving horizontally in a stratified fluid. J. Fluid Mech. 254, 1-21.

Cianferra, M., Ianniello, S., Armenio, V., 2017. Assessment of methodologies for the solution of the Ffowcs Williams and Hawkings equation using les of incompressible single-phase flow around a finite-size square cylinder. submitted.

Cintolesi, C., Petronio, A., Armenio, V., 2015. Large eddy simulation of turbulent buoyant flow in a confined cavity with conjugate heat transfer. Phys. Fluids 27 . http://dx.doi. 
org $/ 10.1063 / 1.4930815$.

DiFrancescantonio, P., 1997. A new boundary integral formulation for the prediction of sound radiation. J. Sound Vib. 202 (4), 491-509.

Farassat, F., Brentner, K.S., 1988. Uses and abuses of the acoustic analogy in helicopter rotor noise prediction. J. Am. Helicopter Soc. 33 (1), 29-36.

Farassat, F., Brentner, K.S., 2003. A modeling aerodynamically generated sound of helicopter rotors. Prog. Aerosp. Sci. 39, 83-120.

Ffowcs-Williams, J., Hawkings, D., 1969. Sound generation by turbulence and surfaces in arbitrary motion. Philos. Trans. R. Soc. 264 (A1151), 321-342.

Gloerfelt, X., Perot, F., Bailly, C., Juve, D., 2005. Flow-induced cylinder noise formulated as a diffraction problem for low mach numbers. J. Sound Vib. 287, 129-151.

Ianniello, S., Muscari, R., Mascio, A.D., 2013. Ship underwater noise assessment by the acoustic analogy.part i: nonlinear analysis of a marine propeller in a auniform flow. J. Mar. Sci. Technol. 18 (4), 547-570.

Inoue, O., Hatakeyama, N., 2002. Sound generation by a two-dimensional circular cylinder in a uniform flow. J. Fluid Mech. 471, 285-314.

Kim, H., Durbin, P., 1988. Observations of the frequencies in a sphere wake and of drag increase by acoustic excitation. Phys. Fluids 31 (11), 3260-3265.

Li, D., Hallander, J., Johansson, T., Karlsson, R., 2015. Cavitation dynamics and underwater radiated noise signature of a ship with a cavitating propeller. VI International Conference on Computational Methods in Marine Engineering.

Lidtke, A., Humphrey, V., Turnock, S., 2015. Feasibility study into a computational approach for marine propeller noise and cavitation modelling. Ocean Eng. ISSN 0029-8018

Lidtke, A., Turnock, S., Humphrey, V., 2016. Characterisation of sheet cavity noise of hydrofoil using the ffowcs williams-hawkings acoustic analogy. Comput. Fluids 130, 8-23.
Lockard, D., 2002. A comparison of ffowcs williams-hawkings solvers for airframe noise applications. AIAA Paper 2002-2580 8th AIAA/CEAS Aeroacoustics Conference, June 17-19.

Lockard, D., Khorrami, M., Choudhari, M., Hutcheson, F., Brooks, T., 2007. Tandem cylinder noise predictions. 13th AIAA/CEAS Aeroacoustics Conference.

Marsden, O., Bogey, C., Bailly, C., 2008. Direct noise computation of the turbulent flow around a zero-incidence airfoil. AIAA J. 46 (4), 874-883.

Meneveau, C., Lund, T., Cabot, W., 1996. A lagrangian dynamic sungrid-scale model of turbulence. J. Fluid Mech. 319, 353-385.

Murphy, E., King, E., 2014. Environmental Noise Pollution. Elsevier.

Najafi-Yazdi, A., Bres, G.A., Mongeau, L., 2011. An acoustic analogy formulation for moving sources in uniformly moving media. Proc. R. Soc. Lond. A467, 144-165.

Nitzkorski, Z., Mahesh, K., 2014. A dynamic technique for sound computation using the Ffowcs Williams and Hawkings equations. Phys. Fluids 26 (11), 115101.

Pando, M.D., Schmid, P., Sipp, D., 2014. A global analysis of tonal noise in flows around aerofoils. J. Fluid Mech. 754, 5-38.

Piomelli, U., Craig, L.S., Sarkar, S., 1997. On the computation of sound by large-eddy simulation. J. Eng. Math. 32, 217-236.

Rodriguez, I., Lehmkuhl, O., Borrell, R., Oliva, A., Perez-Segarra, C., 2010. Direct numerical simulation of turbulent wakes:flow past a sphere at re $=5000$. V European Conference on Computational Fluid Dynamics.

Sakamoto, H., Haniu, H., 1990. A study on vortex shedding from spheres in a uniform flow. J. Fluids Eng. 112, 386-392.

Seidl, V., Muzaferija, S., Peric, M., 1998. Parallel dns with local grid refinement. Appl. Sci. Res. 59, 379-394.

Seror, C., Sagaut, P., Bailly, C., Juvé, D., 2000. On the radiated noise computed by largeeddy simulation. Phys. Fluids 13 (2). 